\title{
Long-term outcomes of vaginal mesh versus native tissue repair for anterior vaginal wall prolapse
}

\author{
Michele Jonsson Funk, \\ Department of Epidemiology, Gillings School of Global Public Health, Chapel Hill, USA. Center \\ for Women's Health Research, University of North Carolina at Chapel Hill, Chapel Hill, NC, USA
}

Anthony G. Visco, Division of Urogynecology, Department of Obstetrics and Gynecology, Duke University, Durham, NC, USA

\author{
Alison C. Weidner, \\ Division of Urogynecology, Department of Obstetrics and Gynecology, Duke University, Durham, \\ NC, USA \\ Virginia Pate, and \\ Department of Epidemiology, Gillings School of Global Public Health, Chapel Hill, USA \\ Jennifer M. Wu \\ Division of Urogynecology, Department of Obstetrics and Gynecology, Duke University, Durham, \\ NC, USA. Duke University Medical Center, Box 3192, 5324 McFarland Avenue, Suite 310, \\ Durham, NC 27707, USA \\ Jennifer M. Wu: jennifer.wu@duke.edu
}

\section{Abstract}

Introduction and hypothesis-To estimate the risk of repeat surgery for recurrent prolapse or mesh removal after vaginal mesh versus native tissue repair for anterior vaginal wall prolapse.

\begin{abstract}
Methods-We utilized longitudinal, adjudicated, healthcare claims from 2005 to 2010 to identify women $\geq 18$ years who underwent an anterior colporrhaphy (CPT 57420) with or without concurrent vaginal mesh (CPT 57267). The primary outcome was repeat surgery for anterior or apical prolapse or for mesh removal/revision; these outcomes were also analyzed separately. We utilized Kaplan-Meier curves to estimate the cumulative risk of each outcome after vaginal mesh versus native tissue repair. Cox proportional hazards models were used to estimate the hazard ratio (HR) for vaginal mesh versus native tissue repair, adjusted for age, concurrent hysterectomy, and concurrent or recent sling.
\end{abstract}

Results-We identified 27,809 anterior prolapse surgeries with 49,658 person-years of followup. Of those, 6,871 (24.7\%) included vaginal mesh. The 5-year cumulative risk of any repeat surgery was significantly higher for vaginal mesh versus native tissue $(15.2 \%$ vs $9.8 \%$, $p<0.0001$ ) with a 5-year risk of mesh revision/removal of 5.9\%. The 5-year risk of surgery for recurrent prolapse was similar between vaginal mesh and native tissue groups (10.4\% vs $9.3 \%$, $p=0.70$. The results of the adjusted Cox model were similar (HR 0.93, 95\% CI: $0.83,1.05$ ).

(C) The International Urogynecological Association 2013

Correspondence to: Jennifer M. Wu, jennifer.wu@duke.edu.

Presented as an oral presentation at the 33rd Annual Meeting of the American Urogynecologic Society, Chicago, IL, USA, 3-6 October 2012.

Conflicts of interest None. 
Conclusions-The use of mesh for anterior prolapse was associated with an increased risk of any repeat surgery, which was driven by surgery for mesh removal. Native tissue and vaginal mesh surgery had similar 5-year risks for surgery for recurrent prolapse.

\section{Keywords}

Anterior colporrhaphy; Pelvic organ prolapse; Surgery; Recurrent prolapse; Vaginal mesh

\section{Introduction}

The recent US Food and Drug Administration (FDA) safety communication on serious complications from transvaginal mesh [1] heightened awareness of graft augmentation in pelvic organ prolapse procedures among healthcare providers, patients, and the general public [2]. The FDA highlighted complications due to surgical mesh devices such as mesh exposure/extrusion, vaginal scarring/shrinkage, pain, dyspareunia, infection, and organ perforation, as well as the repeat surgeries associated with these complications [3]. A rationale for synthetic mesh augmentation was to address the risk of recurrent prolapse after surgical repair, which has been reported to be as high as $29 \%$ [4]. However, the literature suggests that long-term outcomes with vaginal mesh augmentation may not be better than those with native tissue, especially for surgeries in the apical and posterior compartment of the vagina [3].

Prolapse in the anterior compartment is particularly challenging as the short-term recurrence after anterior colporrhaphy is relatively high [5-8]. Randomized trials of native tissue repair versus mesh augmentation have shown that anatomical cure is higher for mesh procedures $[5,6,9]$, and the FDA supports this claim in their systematic review [3]. However, the FDA also noted that "this anatomic benefit may not result in superior symptomatic outcomes or lower rates of repeat surgery for recurrent prolapse" compared with traditional native tissue repair without mesh $[1,3]$. Furthermore, the use of synthetic mesh may be associated with mesh complications, which may require additional interventions and surgical repair. A major limitation of the existing literature is that the cohorts studied were relatively small and the length of follow-up was relatively short. In addition, limited data exist regarding the rate of repeat surgery for recurrent prolapse or mesh complications.

Healthcare claims data of commercially insured patients may provide particularly useful information to address this timely and controversial issue, since a large cohort of women can be evaluated with longer-term follow-up. Furthermore, limitations due to loss to follow-up in trials may be mitigated as these data are based on insurance claims. Thus, the objective of this study was to utilize a large, population-based database to estimate the rate of repeat surgery after vaginal mesh versus native tissue repair for anterior vaginal wall prolapse. We also sought to estimate the rates of repeat surgeries due to recurrent prolapse versus mesh complications and to assess predictors of repeat surgery.

\section{Materials and methods}

\section{Data source}

For this analysis, we utilized 6 years of data (2005-2010) from the MarketScan ${ }^{\circledR}$ Commercial Claims and Encounters $(C C \& E)$ database and Medicare Supplemental and Coordination of Benefits database (copyright () 2011 Thomson Healthcare Incorporated Inc. All rights reserved) [10]. The available data included adjudicated, paid healthcare claims for approximately 28.3 million individuals in 2005, increasing to 48.8 million in 2010. Contributing individuals included those with commercial, employment-based insurance, such as employees, their spouses, dependants, as well as retirees. Of note, in 2010, $55.3 \%$ of 
the US population, or 170.7 million individuals, had employment-based insurance [11]. These de-identified, individual-level inpatient and out-patient claims were aggregated from approximately 100 payers in the United States. Claims and enrollment data were validated by Thomson Reuters to ensure completeness, accuracy, and reliability. Although the data were anonymized, unique individuals can be followed over time using encrypted identification numbers, and detailed enrollment data ensured that only those individuals who could generate a claim were considered as part of the population at risk at any given time. This study was determined to be exempt from further review by the Institutional Review Board at the University of North Carolina at Chapel Hill.

\section{Inclusion criteria and index surgery}

The population at risk included all women aged 18 years and older from 2005 to 2010. Among these women, our goal was to compare women who underwent a native tissue anterior colporrhaphy with those who had an anterior colporrhaphy with mesh augmentation, or vaginal mesh. We identified anterior colporrhaphies based on current procedural terminology (CPT) code 57240 (Table 1). If CPT 57267 (insertion of mesh or other prosthesis for repair of a pelvic floor defect, each site [anterior, posterior compartment], vaginal approach) was present, we counted these surgeries as a vaginal mesh procedures. If CPT 57267 was not present, these surgeries were counted as a native tissue repairs.

In order to assess baseline covariates, including recent urogynecological procedures, we excluded women who did not have at least 6 months of continuous enrollment prior to the first, or index, procedure. We also excluded women who had mesh placed during the baseline period, in order to limit misclassification of our primary exposure, vaginal mesh, and to enable us to attribute future surgery for mesh complications to the index procedure and not prior surgeries. Based on this same rationale, we also excluded women with a prior abdominal or laparoscopic sacrocolpopexy. Similarly, if a procedure to remove or revise mesh (CPT codes 57415, 57426, 57295, and 57296) was performed prior to the index surgery, those women were also excluded (Table 1).

Because the code for mesh insertion, CPT 57267, is not linked to a specific procedure and we wanted to specifically estimate the impact of mesh augmentation for anterior colporrhaphy, we excluded women with other concomitant prolapse procedures, including posterior colporrhaphy (Table 1). For example, if CPT 57267 is listed along with CPT 57260 for an anterior and posterior colporrhaphy, it is impossible to determine if the mesh was placed in the anterior or posterior compartment. Thus, the only way to ensure that mesh was placed in the anterior compartment was to exclude women who underwent other prolapse procedures. Although we excluded concurrent prolapse procedures based on CPT codes (Table 1), we did not exclude concurrent hysterectomy or concurrent sling. Because a sling may also have an impact on the risk of recurrent prolapse, we included both a concurrent sling and any recent sling in the prior 6 months in our definition of a sling procedure.

\section{Repeat surgery}

After the initial anterior colporrhaphy, either with native tissue or vaginal mesh, we identified any repeat surgery for either recurrent anterior or apical prolapse, or a mesh complication. We defined repeat surgery for recurrent prolapse based on subsequent procedures for anterior prolapse (CPT codes $57240,57260,57265,57284,57285,57423$ ) or for apical prolapse (CPT codes 57280, 57282, 57283, 57425). Our rationale for including both anterior and apical procedures was that surgeons may opt for either an anterior and/or apical procedure to manage short-term recurrent anterior vaginal wall prolapse. We defined 
surgery for mesh complications based on the following CPT codes: 57415 removal of vaginal foreign body, 57426 revision/removal of vaginal prosthetic graft laparoscopy, 57295 revise vaginal graft via vaginal approach, and 57296 revise vaginal graft via abdominal approach. It is important to note that repeat surgeries for recurrent prolapse and for mesh complications were not mutually exclusive. In addition, if a surgery for a mesh complication occurred prior to a surgery for recurrent prolapse, we opted to censor individuals at the time of the mesh surgery for the analysis of recurrent prolapse. Similarly, in the analysis for mesh complications, we censored individuals who underwent surgery for recurrent prolapse if it occurred prior to the mesh complication.

For surgery for mesh complications, we evaluated this outcome in both the native tissue and the vaginal mesh cohorts, even though mesh complications should not occur in those undergoing a native tissue repair. It is possible that a mesh complication could occur in the "native tissue" cohort if the patient had mesh placed before the baseline period (more than 6 months prior to the index procedure) or if a midurethral sling resulted in a sling revision/ removal due to a mesh complication that was not coded using the typical CPT code for sling revision/removal.

\section{Statistical analysis}

Using Kaplan-Meier survival curves, we estimated the cumulative risk of repeat surgery for recurrent prolapse or mesh complications overall, and for each outcome separately at yearly intervals. We also estimated the cumulative risk at 2-year follow-up for specific subgroups based on age, calendar year of the index procedure, concomitant or recent sling, and concomitant hysterectomy.

In order to adjust for differences in age, concomitant hysterectomy, or concomitant/recent sling between patients receiving native tissue and those receiving vaginal mesh, we estimated adjusted hazard ratios (HR) using Cox proportional hazards model. We included age as a six-level categorical variable (18-34, 35-44, 45-54, 55-64, 65-74, 75+). Wald Chisquared statistics were used to test the significance of independent predictors with a twosided alpha $=0.05$. Analyses were performed using SAS, v9.2 (SAS Institute, Cary, NC, USA).

\section{Results}

We identified 27,809 anterior prolapse surgeries with 49,658 person-years of follow-up among women aged $\geq 18$ years. Of these, $20,938(75.3 \%)$ were native tissue repairs while $6,871(24.7 \%)$ included vaginal mesh. Women who underwent native tissue repair were younger (median 55 years, interquartile range (IQR) 47, 63) compared with the vaginal mesh surgery (median 59 years, IQR 52, 67, $p<0.0001$ ); Table 2). The median duration of follow-up was similar in those receiving native tissue $(1.3$ years [IQR $0.5,2.7])$ and vaginal mesh (1.4 years [IQR 0.5, 2.7], $p=0.44$ ). When evaluating the calendar year in which the index surgery was performed, there were differences between groups with an increase in the proportion of procedures involving vaginal mesh from 2005 to $2010(p<0.0001)$. Fewer native tissue repairs had a concomitant or recent sling $(62.4 \%$ vs $70.6 \%, p<0.0001$, but a higher proportion underwent a concurrent hysterectomy ( $38.3 \%$ vs $18.4 \%, p<0.0001$; Table 2).

When we estimated the risk of any repeat surgery after the index surgery for anterior prolapse, the 5 -year cumulative risk was significantly higher for vaginal mesh ( $15.2 \%$ vs $9.8 \%, p<0.0001)$. However, the 5-year cumulative risk of recurrent prolapse surgery was similar in the two groups (native tissue: $9.3 \%$ [95\% CI 8.6, 10.0]; vaginal mesh: $10.4 \%$ [95\% CI 8.8, 12.1] $p=0.70$; Table 3). The difference in the risk of any repeat surgery was due 
to the significantly higher 5-year risk of mesh complication in the vaginal mesh group (5.9 $\%$ [95\% CI 5.0, 6.9] compared with native tissue $(0.7 \%$ [95\% CI 0.5, 0.9], $p<0.0001)$.

In order to further evaluate the impact of age, year of index surgery, and concurrent hysterectomy or sling, we estimated the 2-year cumulative risks of repeat surgery for recurrent prolapse and mesh complications in these subgroups of patients. These 2-year estimates are shown in Table 4 as absolute risks with 95\%CI. Age at index surgery or year of the index surgery did not yield a definitive trend for surgery for recurrent prolapse at 2 years post-operatively for either the native tissue or the vaginal mesh cohorts. However, concurrent hysterectomy decreased the risk of surgery for recurrent prolapse in the native tissue group $(4.4 \%$ [95\% CI 3.9, 4.9] versus $7.8 \%$ [95\% CI 7.3, 8.4]) and recent/concurrent sling decreased surgery for recurrent prolapse in both groups (Table 4).

Surgery for a mesh complication was primarily pertinent to the vaginal mesh cohort. There was no consistent trend for mesh surgery based on age, concurrent hysterectomy or recent/ concurrent sling. However, the 2-year risk of mesh surgery increased from $2.6 \%$ (95\% CI $0.9,4.3)$ in 2005 to $4.8 \%(95 \%$ CI $3.6,6.0)$ in 2008.

We evaluated the risk of any repeat surgery as well as surgery for recurrent prolapse and a mesh complication using Cox proportional hazards models adjusted for age, concurrent hysterectomy, and concurrent or recent sling. Vaginal mesh was associated with an increase in the risk of any repeat surgery compared with native tissue (HR 1.33, 95\% CI 1.20, 1.46). The risk of surgery for recurrent prolapse was similar in those with vaginal mesh versus native tissue (HR 0.93, 95\% CI 0.83, 1.05). Thus, the difference in the rate of repeat surgeries was driven by mesh complications (HR 8.21, 95\%CI 6.31, 10.67).

\section{Discussion}

In this population-based cohort of 27,809 vaginal procedures for anterior vaginal wall prolapse, the 5-year cumulative risk of repeat surgery was higher for vaginal mesh procedures $(15.2 \%)$ than for native tissue surgeries $(9.8 \%)$, which was due to subsequent surgeries for mesh removal and not for recurrent prolapse. These estimates are useful in counseling patients regarding the need for reoperation after prolapse surgery.

The 2011 FDA update regarding transvaginal mesh reported that mesh augmentation may provide anatomical benefit in the anterior compartment, but with no improvement in the quality of life [1]. This claim was supported by the initial reports of several randomized trials that found that prolapse procedures utilizing mesh had a lower risk of recurrent prolapse $[5,6,9,12,13]$. Systematic reviews and meta-analyses also supported this conclusion $[7,8,14,15]$. However, limitations of these existing trials included a nonblinded evaluator [3] and a relatively short follow-up of 12 months [5, 9, 13, 16-18]. Furthermore, there was some evidence that any improvement in anatomical outcomes from the use of mesh was of little benefit to the patient, since the few studies that assessed patient-centered outcomes, such as quality of life, failed to show a difference between native tissue and mesh augmentation procedures [12, 14, 17].

While the risk of anatomical recurrence may be lower with mesh augmentation in the anterior compartment, a critical question is whether mesh decreases the need for reoperation for recurrent prolapse. Nieminen et al. reported that $18 \%$ (17 out of 96 ) in the native tissue and $11 \%$ (11 out of 104) in the mesh group were referred for reoperation for either recurrent prolapse or urinary incontinence by 3 years, a difference that was not statistically significant [6]. In our study, we found that the risk of repeat surgery for prolapse was comparable at 9.3 $\%$ for native tissue versus $10.4 \%$ for vaginal mesh by 5 years. Adjusting for differences in 
age and other concurrent pelvic organ procedures (hysterectomy and sling) did not meaningfully alter the observed association.

In our analysis, the driving factor for a higher risk of reoperation after mesh augmentation was the need for surgery for a mesh complication. Randomized trials have reported a risk of mesh exposure/extrusion that ranges from $5 \%$ to $19 \%[6,9,12,17,18]$. However, some of these mesh exposures/extrusions were managed conservatively, and the proportion of subjects undergoing reoperation in an operating room for mesh complications was approximately $3.2 \%$ to $7.6 \%[5,6,12,13]$. In an analysis of over 800 prolapse procedures using implanted grafts or mesh, Nguyen et al. reported that the reoperation risk for vaginal mesh exposure was $3.4 \%$ during a 21 -month period [19]. These data were comparable to estimates in this report of $3.0 \%$ risk of mesh exposure surgery at 1 year, increasing to $5.9 \%$ at 5 years.

We analyzed predictors of future surgery and found that concurrent hysterectomy and recent/concurrent sling decreased the risk of surgery for recurrent anterior and/or apical prolapse. One explanation is that if uterine prolapse was present, a hysterectomy decreased persistent or recurrent prolapse in the apical compartment. A prior or concurrent sling may also decrease future anterior prolapse given the supportive nature of a sling in the distal anterior compartment. Regarding predictors for surgery for mesh complications, we found that the risk of reoperation was lower for surgeries performed in 2005 and that this risk increased each subsequent year. It is possible that more recently available mesh materials and/or kits may be associated with a higher risk of mesh complications. Another possibility is that less experienced providers began to perform these procedures and had higher complication rates.

The strengths of this study were based in the inherent characteristics of a large, populationbased database, which allowed us to evaluate over 20,000 native tissue and 6,800 vaginal mesh procedures for anterior vaginal wall prolapse. These healthcare claims data allowed us to follow patients over time, even if they did not return to their primary surgeon or the hospital of their index surgery for their reoperation. This was an important issue because patients with a complication may seek care from another surgeon at a different institution. Insurance claims data enabled us to capture any reoperations, as long as a patient remained insured. By utilizing data from 2005 to 2010, we were able to provide 5-year cumulative risks, which is significantly longer than the follow-up periods of existing studies.

This study was limited by constraints inherent in the database. This database represents those with health insurance, and thus, the results may not be generalizable to an uninsured population. In addition, data regarding race, physical examination findings, such as body mass index and severity of prolapse, medical history and surgical data were not available, and we were unable to evaluate the impact of these variables on our outcomes. Because we did not have all of the baseline characteristics of the study population, it was possible that a higher proportion of women who underwent vaginal mesh repair had recurrent or more severe prolapse compared with women in the native tissue group. However, given the fact that $\sim 75,000$ vaginal mesh procedures were performed in 2010 [3], it was unlikely that mesh was only utilized in women with recurrent prolapse.

As with any secondary data analysis, misclassification may have existed. For example, a small number of women in the native tissue group had mesh complications. We suspect that these women had mesh placed prior to the baseline period and/or had a different type of procedure that involved mesh, such as a sling. Another limitation was that we cannot determine the specific mesh or mesh kit/procedure that was performed, and therefore cannot comment on whether different mesh procedures carry different risks of complications. 
Because of limitations imposed by the CPT coding system, which was central to our database, we were forced to exclude certain women from our analysis, in order to limit misclassification of our treatment groups to the greatest extent possible, and to define a more homogeneous cohort, overall, in which the effect of mesh could be evaluated. We have no reason to believe, however, that these a priori exclusions affected either study group preferentially.

Despite these limitations, our study provided insight into the longer-term risk of surgical intervention for recurrent prolapse and mesh complications after native tissue versus vaginal mesh for anterior vaginal wall prolapse. These estimates are valuable in counseling patients regarding what they can anticipate for reoperation rates for recurrent pro-lapse and/or mesh complications. Our results clearly indicated that patients seeking to minimize the risk of future surgery should carefully consider mesh augmentation of the anterior compartment, since the overall risk of future surgery was actually higher in the women receiving mesh. Furthermore, the rate of surgery for recurrent prolapse was no different with or without mesh. Lastly, our findings emphasize the importance of prospective, comprehensive, and long-term assessments of the safety and effectiveness of transvaginal mesh for pelvic organ prolapse.

\section{Acknowledgments}

Funding This project was supported by grant number K02HS017950 from the Agency for Healthcare Research and Quality (AHRQ; to M.J.F) and grant number K23HD068404 from the Eunice Kennedy Shriver National Institute of Child Health \& Human Development (to J.M.W). The content is solely the responsibility of the authors and does not necessarily represent the official views of the Agency for Healthcare Research and Quality.

\section{References}

1. FDA Safety Communication. [Accessed on 12 June 2012] Update on serious complications associated with transvaginal placement of surgical mesh for pelvic organ prolapse. Issued on 13 July 2011. http://www.fda.gov/medicaldevices/safety/alertsandnotices/ucm262435.htm

2. [Accessed on 9 July 2012] Dangerous medical devices: most medical implants have never been tested for safety. Consumer Reports Magazine. May. 2012 at: http://www.consumerreports.org/cro/ magazine/2012/04/cr-investigates-dangerous-medical-devices/index.htm

3. [Accessed on 12 June 2012] FDA Executive Summary: Surgical mesh for the treatment of women with pelvic organ prolapse and stress urinary incontinence. Sep 8-9. 2011 http://www.fda.gov/ downloads/AdvisoryCommittees/CommitteesMeetingMaterials/MedicalDevices/ MedicalDevicesAdvisoryCommittee/ObstetricsandGynecologyDevices/UCM270402.pdf

4. Olsen AL, Smith VJ, Bergstrom JO, Colling JC, Clark AL. Epidemiology of surgically managed pelvic organ prolapse and urinary incontinence. Obstet Gynecol. 1997; 89(4):501-506. [PubMed: 9083302]

5. Altman D, Vayrynen T, Engh ME, Axelsen S, Falconer C. Anterior colporrhaphy versus transvaginal mesh for pelvic-organ prolapse. N Engl J Med. 2011; 364(19):1826-1836. [PubMed: 21561348]

6. Nieminen K, Hiltunen R, Takala T, et al. Outcomes after anterior vaginal wall repair with mesh: a randomized, controlled trial with a 3 year follow-up. Am J Obstet Gynecol. 2010; 203(3):235, e1e8. [PubMed: 20494332]

7. Foon R, Toozs-Hobson P, Latthe PM. Adjuvant materials in anterior vaginal wall prolapse surgery: a systematic review of effectiveness and complications. Int Urogynecol J Pelvic Floor Dysfunct. 2008; 19(12):1697-1706. [PubMed: 18607524]

8. Jia X, Glazener C, Mowatt G, et al. Efficacy and safety of using mesh or grafts in surgery for anterior and/or posterior vaginal wall prolapse: systematic review and meta-analysis. BJOG. 2008; 115(11):1350-1361. [PubMed: 18715243]

9. Nguyen JN, Burchette RJ. Outcome after anterior vaginal prolapse repair: a randomized controlled trial. Obstet Gynecol. 2008; 111(4):891-898. [PubMed: 18378748] 
10. [Accessed on 12 June 2012] Thomson Reuters MarketScan ${ }^{\circledR}$ Commercial Claims and Encounters Database, 2000-2009. Source: RED BOOK ${ }^{\circledR}$ and Market-Scan ${ }^{\circledR}$ are registered trademarks of Thomson Reuters (Healthcare) Inc. http://thomsonreuters.com/products_services/healthcare/ healthcare_products/pharmaceuticals/mktscan_res_db/

11. DeNavas-Walt, C.; Proctor, BD.; Smith, JC. US Census Bureau, Current Population Reports, P60239, Income, Poverty, and Health Insurance Coverage in the United States: 2010. U.S. Government Printing Office; Washington, D.C: 2011. http://www.census.gov/prod/2011pubs/ p60-239.pdf [Accessed on 24 June 2012]

12. Menefee SA, Dyer KY, Lukacz ES, Simsiman AJ, Luber KM, Nguyen JN. Colporrhaphy compared with mesh or graft-reinforced vaginal paravaginal repair for anterior vaginal wall prolapse: a randomized controlled trial. Obstet Gynecol. 2011; 118(6):1337-1344. [PubMed: 22067717]

13. Withagen MI, Milani AL, den Boon J, Vervest HA, Vierhout ME. Trocar-guided mesh compared with conventional vaginal repair in recurrent prolapse: a randomized controlled trial. Obstet Gynecol. 2011; 117(2 Pt 1):242-250. [PubMed: 21252735]

14. Maher CM, Feiner B, Baessler K, Glazener CM. Surgical management of pelvic organ prolapse in women: the updated summary version Cochrane review. Int Urogynecol J. 2011; 22(11):14451457. [PubMed: 21927941]

15. Sung VW, Rogers RG, Schaffer JI, et al. Graft use in transvaginal pelvic organ prolapse repair: a systematic review. Obstet Gynecol. 2008; 112(5):1131-1142. [PubMed: 18978116]

16. Hiltunen R, Nieminen K, Takala T, et al. Low-weight poly-propylene mesh for anterior vaginal wall prolapse: a randomized controlled trial. Obstet Gynecol. 2007; 110(2 Pt 2):455-462. [PubMed: 17666627]

17. Carey M, Higgs P, Goh J, et al. Vaginal repair with mesh versus colporrhaphy for prolapse: a randomised controlled trial. BJOG. 2009; 116(10):1380-1386. [PubMed: 19583714]

18. Lunardelli JL, Auge AP, Lemos NL, et al. Polypropylene mesh vs. site-specific repair in the treatment of anterior vaginal wall prolapse: preliminary results of a randomized clinical trial. Rev Col Bras Cir. 2009; 36(3):210-216. [PubMed: 20076900]

19. Nguyen JN, Jakus-Waldman SM, Walter AJ, White T, Menefee SA. Perioperative complications and reoperations after incontinence and prolapse surgeries using prosthetic implants. Obstet Gynecol. 2012; 119(3):539-546. [PubMed: 22353951] 


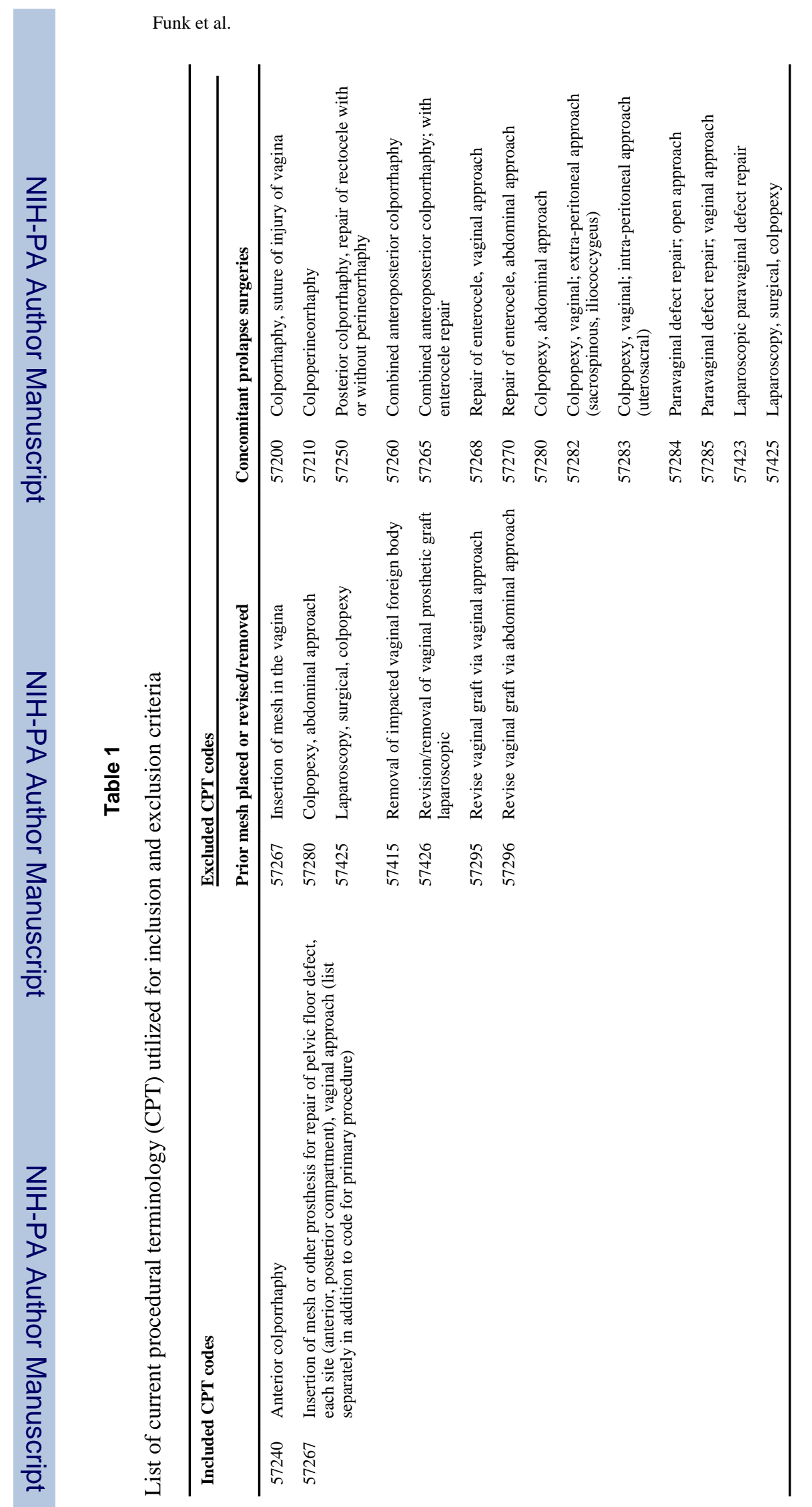

Page 9 


\section{Table 2}

Characteristics of women aged $\geq 18$ undergoing vaginal mesh versus native tissue repair for anterior vaginal wall prolapse from 2005 to 2010

\begin{tabular}{|c|c|c|c|}
\hline & Native tissue $(n=20,938)$ & Vaginal mesh $(n=6,871)$ & $p$ value \\
\hline \multicolumn{4}{|l|}{ Age } \\
\hline Median (years) & 55 (IQR 47, 63) & 59 (IQR 52, 67) & $<0.0001$ \\
\hline Range (years) & $19-93$ & $22-95$ & \\
\hline \multicolumn{4}{|l|}{ Age } \\
\hline $18-34$ & $668(3.2)$ & $84(1.2)$ & \\
\hline $35-44$ & $3,262(15.6)$ & $593(8.6)$ & \\
\hline $45-54$ & $6,027(28.8)$ & $1,591(23.2)$ & \\
\hline $55-64$ & $6,594(31.5)$ & $2,503(36.4)$ & \\
\hline $65-74$ & $2,784(13.3)$ & $1,262(18.4)$ & \\
\hline $75+$ & $1,603(7.7)$ & $838(12.2)$ & \\
\hline \multicolumn{4}{|l|}{ Follow-up time } \\
\hline Median (years) & 1.3 (IQR $0.5,2.7$ ) & 1.4 (IQR 0.5, 2.7) & 0.44 \\
\hline \multicolumn{4}{|l|}{ Calendar year } \\
\hline 2005 & $3,214(15.4)$ & $563(8.2)$ & $<0.0001$ \\
\hline 2006 & $2,968(14.2)$ & $923(13.4)$ & \\
\hline 2007 & $3,145(15.0)$ & $1,175(17.1)$ & \\
\hline 2008 & $3,652(17.4)$ & $1,497(21.8)$ & \\
\hline 2009 & $4,197(20.0)$ & $1.424(20.7)$ & \\
\hline 2010 & $3,762(18.0)$ & $1,289(18.8)$ & \\
\hline \multicolumn{4}{|l|}{ Concomitant surgeries } \\
\hline Recent/concurrent sling & $13,061(62.4)$ & 4,849 (70.6) & $<0.0001$ \\
\hline Concurrent hysterectomy & $8,018(38.3)$ & $1,263(18.4)$ & $<0.0001$ \\
\hline
\end{tabular}




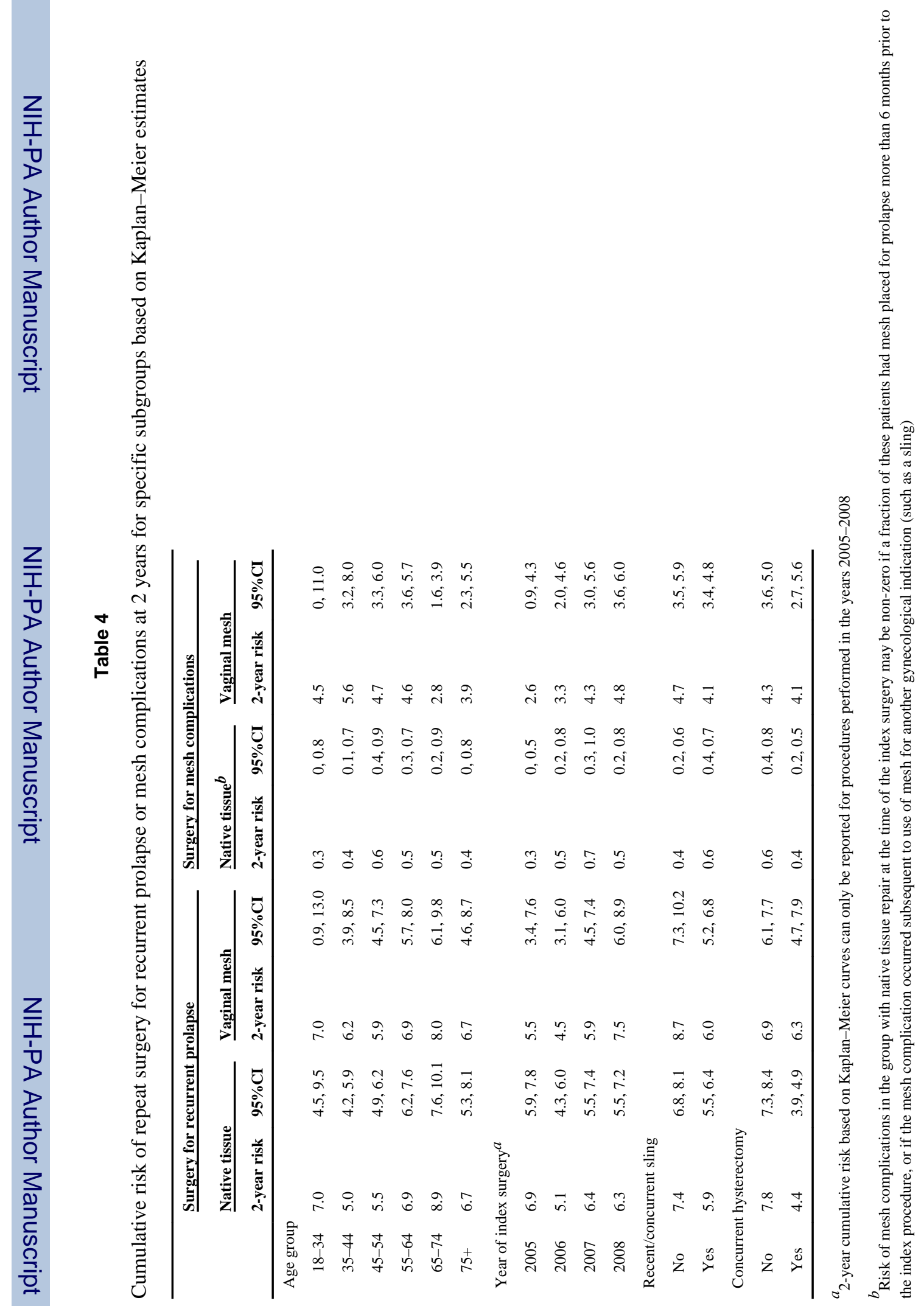

